\title{
INTEGRAL FORMULAS ON PROJECTIVE SPACE AND THE RADON TRANSFORM OF GINDIKIN-HENKIN-POLYAKOV
}

\author{
BO BERNDTSSON
}

\begin{abstract}
We construct a variant of Koppelman's formula for $(0, q)$-forms with values in a line bundle, $O(l)$, on projective space. The formula is then applied to a study of a Radon transform for $(0, q)$-forms, introduced by Gindikin-Henkin-Polyakov. Our presentation follows along the basic lines of Henkin-Polyakov [3], with some simplifications.
\end{abstract}

\section{Introduction}

In two papers $([2]$ and $[3])$, Gindikin-Henkin, and Henkin-Polyakov have developed a theory of a Radon transform for differential forms on domains in projective space. The two basic results are that the Radon transform defines an injective map on the Dolbeault cohomology groups (i.e. that the Radon transform of a $\bar{\partial}$-closed differential form is zero if and only if the form is $\bar{\partial}-$ exact), and an inversion formula which, among other things, gives a description of the image of the map. As a matter of fact $[2]$ and $[3]$ give two different inversion formulas, of which the latter works in greater generality and at first looks quite different from the earlier one.

The aim of this paper is to give proofs of those results using weighted integral formulas (cf.[1]). This is in no way radically different from the methods in [2] and $[\mathbf{3}]$, but in our view it leads to some simplifications. Let us explain briefly what they consist in.

The starting point in [3] is a "Koppelman's formula" which represents a $\bar{\partial}$-closed form, $f$, as

$$
f=\bar{\partial} K(f)+R(f)
$$

so that $\bar{\partial} K(f)$ is "the $\bar{\partial}$-exact part" and $R(f)$ is a remainder which vanishes if $f$ is $\bar{\partial}$-exact. Then one goes on to show that $R(f)$ can be related to the Radon transform. Henkin and Polyakov obtain this descomposition by lifting the form $f$ to (the sphere in) $\mathbb{C}^{n+1}$, by means of the projection map

$$
\pi: \mathbf{C}^{n+1} \rightarrow \mathbf{P}^{n}
$$


and then using a classical formula in $\mathbb{C}^{n+1}$. Instead of this we will construct an explicit kernel directly on $\mathbf{P}^{n}$. What this amounts to is that we don't need to integrate over the fibers of the map $\pi$, which makes our formula simpler and more explicit. As it turns out the freedom to chose weight factor enables us to treat forms with values in the standard line bundles, $O(l)$, on $p^{n}$. From the form of the kernel one can then read off almost directly the definition of the Radon transform. We can also give a rather simple proof of the inversion formula by letting the weight vary. Finally we also note that the different inversion formulas of $[2]$ and $[3]$ can be viewed as coming from different choices of weight.

Once again, it should be stressed that we have no results that are basically new, but that the difference lies mostly in the presentation. Since thus our aim is partly expository we have tried to make the paper as selfcontained as possible, starting with a preliminary section with background material about projective space.

As is the case in $[\mathbf{2}]$ and $[\mathbf{3}]$ our paper deals with forms of bidegree $(0, q)$ or $(n, q)$. The construction of integral kernels on $\boldsymbol{P}^{n}$ in the general case of $(p, q)$ forms is more difficult. In a last section we sketch how a recent idea of $C$. Laurent-Thiebaut and J.P. Demailly can be used here. They showed how one can construct kernels on a complex manifold given a holomorphic connection on a complex vector bundle over the manifold. In their formalism, however, there appear certain "parasitary terms", which depend on the curvature of the connection. It turns out that, in the case of $P^{n}$, the curvature that enters has a particularity simple form, which enables us to push their construction one step further and get rid of the undesirable terms. We have not applied this construction to the problem of the Radon transform, but give it mostly as curiosa.

\section{Preliminaries on $p^{n}(c)$}

$p^{n}(C)$ (or briefly $\left.p^{n}\right)$ is the set of equivalence classes

$$
[z]=\left[z_{0}, \cdots, z_{n}\right], \quad z \neq 0 \text { in } \varsigma^{n+1}
$$

where

$$
[z]=[\omega]
$$

if there is a nonzero complex number $\lambda$ such that

$$
z=\lambda \omega \text {. }
$$

We say that $z=\left(z_{0}, \cdots z_{n}\right)$ are the homogenous coordinates of the point $[z]$. A hyperplane in $\beta^{n}$ is a set of the type

$$
\Pi_{\xi}=\left\{[z]_{i} \sum_{0}^{n} \xi_{j} z_{j}=0\right\}
$$


where $\xi \in \mathbb{C}^{n+1} \backslash\{0\}$. Similary, a q-dimensional plane in $\mathrm{P}^{n}$ is an intersection of $(n-q)$ hyperplanes in general position, i.e. a set of the type

$$
\Pi_{\xi}=\Pi_{\xi^{1}, \ldots, \xi^{n-q}}=\left([z] ; \sum_{0}^{n} \xi_{j}^{m} z_{j}=0 \quad, \quad m=1, \ldots, n-q\right),
$$

where $\left(\xi^{m}\right)$ is linearily independent.

$P^{n}$ is covered by the $(n+1)$ open sets

$$
U_{j}=\left\{[z]_{i} z_{j} \neq 0\right\}
$$

each of which is biholomorphic to $\mathrm{C}^{n}$ under the map

$$
\left[z_{0}, \ldots, z_{n}\right] \rightarrow\left(\frac{z_{0}}{z_{j}}, \ldots, \frac{z_{j-1}}{z_{j}}, \frac{z_{j+1}}{z_{j}}, \ldots, \frac{z_{\mathfrak{n}}}{z_{j}}\right)
$$

The complement of $U_{j}$ in $P^{n}$ is the hyperplane $\left\{|z| ; z_{j}=0\right\}$, sometimes called the hyperplane at infinity (relative to $U_{j}$ ).

For each integer $l$ we have a line bundle, $O(l)$, over $\mathrm{p}^{n}$ defined by the transition functions

$$
g_{j k}=\left(\frac{z_{k}}{z_{j}}\right)^{l} \text { on } U_{j} \cap U_{k} .
$$

Thus a section to $O(l)$ is a function $f^{j}$ on each $U_{j}$ such that

$$
f^{j}=g_{j k} f^{k} \text { on } U_{j} \cap U_{k} .
$$

Given such a section

$$
\tilde{f}\left(z_{0}, \ldots, z_{n}\right)=z_{j}^{l} f^{j}([z])
$$

is a well defined function on $\mathrm{C}^{n+1}$ which is homogenous of degree $l$ in $z$. Conversely the same equation defines a section to $O(l)$ if $\tilde{f}$ is $l$-homogenous in $z$. Hence we may identify sections to $O(l)$ with $l$-homogenous functions on $\mathbb{C}^{n+1}$. In particular a homogenous polynomial of degree $l$ defines a holomorphic section to $O(l)$ if $l \geq 0$.

Next we consider differential forms on (open sets of ) $\mathrm{P}^{n}$. Since

$$
\pi: \mathfrak{C}^{n+1} \rightarrow \mathbf{P}^{n}, \pi(z)=[z]
$$

is surjective, (or rather since $d \pi$ is surjective) the pullback, $\pi^{*}$, of forms on $\mathrm{P}^{n}$ to $C^{n+1}$ is injective. Therefore we can identify a form on $P^{n}$ with its pullback, which simply means that we write the form in homogenous coordinates. If for instance we chose inhomogenous coordinates

$$
\frac{z_{1}}{z_{0}}, \ldots, \frac{z_{n}}{z_{0}}
$$


on $U_{0}$, a $(1,0)$-form, on $U_{0}$ can be written

$$
f=\sum_{1}^{n} f_{j} d \frac{z_{j}}{z_{0}}
$$

where the coefficients $f_{j}$ can be considered as 0-hornogenous functions of $z$. Thus

$$
f=\sum_{1}^{n} f_{j} d \frac{z_{j}}{z_{0}}=\sum_{1}^{n}\left(f_{j} / z_{0}\right) d z_{j}-\frac{1}{z_{0}^{2}}\left(\sum_{1}^{n} z_{j} f_{j}\right) d z_{0}=: \sum_{0}^{n} F_{j} d z_{j} .
$$

Here the coefficients $F_{j}$ satisfy

a) $\sum_{0}^{n} F_{j} z_{j}=0$ and

b) $F_{j}$ are homogenous of degree -1 in $z$.

If, conversely, $F_{j}$ satisfies a) and b) the same relation can be used to define a form on $U_{0}$ and a similar relation works on any $U_{j}$. Hence a) and b) characterize the projective forms of bidegree $(0,1)$, i. e. the forms on $\mathbb{C}^{n+1}$ in the image of $\pi *$. More generally we have:

A form $f$ on $\mathbb{C}^{n+1}$ of bidegree $(p, q)$ is projective if and only if

$$
\left\langle f, \bar{\partial}|z|^{2} \wedge g\right\rangle=0 \quad \text { and } \quad\left\langle f, \partial|z|^{2} \wedge g\right\rangle=0
$$

for all forms $g$, and moreover the coefficients of $f$ are homogenous of degree $(-p,-q)$ in $z$ and $\bar{z}$ respectively.

Here the brackets stand for the scalar product on forms induced by the standard metric on $\mathbb{C}^{n+1}$ (the forms $d z_{I} \wedge d \bar{z}_{J}=d z_{1} \wedge \ldots, d z_{i_{p}} \wedge \ldots, d \bar{z}_{j_{q}}$ form an orthonormal basis on $(p, q)$ forms if $\left(z_{0}, \ldots, z_{n}\right)$ are orthonormal coordinates on $\mathbb{C}^{n+1}$, and forms of different bidegree have scalar product zero).

It is enough to verify this claim at a given point $p \in \mathbb{C}^{n+1}$, and we can choose orthonormal coordinates so that $[p]=[1,0 \ldots, 0]$. We take

$$
w_{i}=\frac{z_{i}}{z_{0}} \quad, \quad i=1, \ldots, n
$$

as inhomogenous coordinates on $\mathrm{P}^{n}$ near $[p]$. At $p$

$$
d w_{i}=\frac{d z_{i}}{z_{0}}
$$

Hence a projective form will not contain $d z_{0}$ or $d \bar{z}_{0}$ which means that (1) holds. If conversely $f$ does not contain $d z_{0}, d \bar{z}_{0}$

$$
f=\sum f_{I J} d z_{I} \wedge d \bar{z}_{J}=\sum\left(f_{I J} z_{0}^{p} z_{0}^{-q}\right) d w_{I} \wedge d \bar{w}_{J}
$$

at $p$ which defines $f$ as a form at $[p]$. 
The operation of interior multiplication is defined by

$$
\langle h\rfloor f, g\rangle=\langle f, \bar{h} \wedge g\rangle
$$

if $f, h, g$ are forms. Thus, we see that if $f$ is projective and $h$ is an arbitrary $(r, s)$ form whose coefficients are homogenous of degree $(s, r)$, then $h] f$ is again projective.

Let us finally study forms on $P^{n}$ with values in $O(l)$. Such a form can be thought of as a scalarvalued form $f^{j}$ on each $U_{j}$ such that

$$
\begin{gathered}
f^{j}=\left(\frac{z_{k}}{z_{j}}\right)^{l} f^{k} \quad \text { on } U_{j} \cap U_{k}, \text { i. e. } \\
z_{j}^{l} f^{j}=z_{k}^{l} f^{k}
\end{gathered}
$$

in homogenous coordinates. So, written in homogenous coordinates a form of bidegree $(1,0)$ with values in $O(l)$ is a form

$$
\sum_{0}^{n} F_{j} d z_{j}
$$

where

a) $\sum_{0}^{n} F_{j} z_{j}=0$ and

b) $F_{j}$ are homogenous of degree $l-1$ in $z$.

On $U_{j}$ we can consider the $(n, 0)$-form

$$
\omega_{j}=(-1)^{j} \underset{i=j}{\wedge} d \frac{z_{i}}{z_{j}} .
$$

This is clearly a nonvanishing $(n, 0)$-form and a simple computation shows that

$$
\omega_{j}=\sum_{0}^{k}(-1)^{k} \frac{d z_{0} \wedge \ldots, d \hat{z}_{k} \wedge \ldots, d z_{n}}{z_{j}^{n+1}} .
$$

Hence

$$
\omega_{j}=\left(\frac{z_{k}}{z_{j}}\right)^{n+1} \omega_{k} \quad \text { on } \quad U_{j} \cap U_{k} .
$$

Since any scalarvalued $(n, 0)$-form $f$ can be written

$$
f=f^{j} \omega_{j} \quad \text { on } \quad U_{j}
$$

where the $f^{j}$ must satisfy

$$
f^{j}=\left(\frac{z_{j}}{z_{k}}\right)^{n+1} f^{k} \quad \text { on } \quad U_{j} \cap U_{k}
$$

we see that the bundle of $(n, 0)$-forms is isomorphic to $O(-(n+1))$. Similary a $(n, q)$-form with values in $O(l)$ can be identified with a $(0, q)$-form with values in $O\left(l^{\prime}\right)$ where

$$
l^{\prime}=l-(n+1) \text {. }
$$




\section{Integral kernels on $P^{n}$}

In this section we will construct integral kernels for the $\bar{\partial}$-operator on domains in $p^{n}$. They will operate on forms of bidegree $(n, q)$ or $(0, q)$ with values in a line bundle $O(l)$, and satisfy a version of Koppelman's formula. In [3], Henkin and Polyakov have obtained such formulas by pulling forms on $P^{n}$ back to the sphere in $C^{n+1}$ using $\pi^{*}$. Here we will work directly on $P^{n}$ writing our kernels in homogenous coordinates. First we recall the construction of weighted integral kernels in $\mathbb{C}^{n}$ (cf.|x]).

Consider a domain $D \subset C^{n}$ with smooth boundary. Let

$$
s=\left(s_{1}, \ldots, s_{n}\right): \bar{D} \times \bar{D} \rightarrow \mathbb{C}^{n}
$$

be a $C^{1}$-function satisfying

$$
|s(\zeta, z)| \leq C|\zeta-z| \text { and }|\langle s, \zeta-z\rangle| \geq c|\zeta-z|^{2}
$$

uniformly for $\zeta \in \bar{D}$ and $z$ in any compact subset of $D$. Here for $\zeta, \eta \in \mathbb{C}^{n}$

$$
\langle\varsigma, \eta\rangle=\sum \varsigma_{j} \eta_{j}
$$

Then let

$$
Q: \bar{D} \times \bar{D} \rightarrow \mathbb{C}^{n}
$$

be any $C^{1}$-function. With $s$ and $Q$ we identify the $(1,0)$-forms

$$
s=\sum_{1}^{n} s_{j} d\left(s_{j}-z_{j}\right), \quad Q=\sum_{1}^{n} Q_{j} d\left(s_{j}-z_{j}\right)
$$

which we denote by the same letters. Let $G(t)$ be an holomorphic function of one complex variable defined in a region such that the following formulas make sense, and which satisfies $G(0)=1$. We then define

$$
\begin{gathered}
K=\sum_{k=0}^{n-1} \frac{(-1)^{k}}{k !} G^{(k)}(\langle Q, \zeta-z\rangle) \frac{s \wedge(\bar{\partial} s)^{n-1-k} \wedge(\bar{\partial} Q)^{k}}{\langle s, \zeta-z\rangle^{n}-k} \\
P=\frac{(-1)^{n+1}}{n !} G^{(n)}(\langle Q, \zeta-z\rangle)(\bar{\partial} Q)^{n}
\end{gathered}
$$

Here $G^{(i)}$ is the $k$-th derivative of $G$, and the $\bar{\partial}$ is taken with respect to $\zeta$ and $z$. Let $K_{p, q}$ be the component of $K$ of bidegree $(p, q)$ in $z$ and $(n-p, n-q-1)$ in $\zeta$, and define $P_{p, q}$ in the same way. We then have the following result (cf. Theorem 1 and Theorem 5 of $[\mathbf{I} \mid)$ : 
Theorem. Let $f$ be $a(p, q)$ form in $C^{1}(\bar{D})$. Then

$$
\begin{aligned}
& f(z)=C_{p, q, n}\left(\int_{\partial D} f(\zeta) \wedge K_{p, q}(\zeta, z)-\int_{D} \bar{\partial} f(\zeta) \wedge K_{p, q}(\zeta, z)+\right. \\
& \left.+(-1)^{p+q+1}\left(\bar{\partial}_{z} \int_{D} f(\zeta) \wedge K_{p, q-1}(\zeta, z)+\int_{D} f(\zeta) \wedge P_{p, q}(\zeta, z)\right)\right) .
\end{aligned}
$$

Here $K_{p,-1}=: 0$.

To be quite accurate the theorem is stated in [x] only under the assumption that $Q(\zeta, z)$ be holomorphic in $z$ in which case $P_{p, q}$ vanishes for $q>0$. However the same proof gives the result as formulated here.

We refer to (3) as Koppelmans formula and our first objective is to generalize the construction to domains in projective space. First we consider the case of $(n, q)$-forms. The construction follows precisely the same pattern as in the case of $\mathfrak{c}^{n}$. We write the kernels in homogenous coordinates, using two differential forms $s$ and $Q$. The novelty is that we must make sure that $s$ and $\bar{\partial} Q$ define projective forms. Therefore we suppose as given a domain $D$ in $\mathrm{F}^{n}$ and two functions

$$
s, Q: \bar{D} \times \bar{D} \rightarrow \epsilon^{n+1}
$$

satisfying:

i) $s(\zeta, z)$ is homogenous of degree -1 in $\zeta$ and 0 in $z$.

ii) $\langle s, z\rangle=\sum_{0}^{n} s_{j} z_{j}=0$.

iii) $|s(\varsigma, z)| \leq C d(\varsigma, z)$ and $|(s, \zeta\rangle| \geq c d^{2}(\zeta, z)$

uniformly for $\zeta \in \bar{D}$ and $z$ in any compact part of $D$. Here $d$ is any distance function that behaves like the euclidean distance in local coordinates (a specific choice will be given later but is unimportant so far).

iv) $Q(\varsigma, z)$ is homogenous of degree -1 in $z$ and 0 in $\zeta$.

v) $\langle Q, z\rangle=1$.

As before we denote also by $s$ and $Q$ the forms

$$
s=\sum_{0}^{k} s_{j} d z_{j} \text { and } Q=\sum_{0}^{n} Q_{j} d z_{j}
$$

(since we are dealing with $(n, q)$-forms we use $d z_{j}$ instead of $d\left(\zeta_{j}-z_{j}\right)$ ). If conditions i)-iii) are fullfflled $s$ is a projective $(1,0)$-form with values in $O(1)$ when considered as a form in $z$ (cf. a) and b) of sec. 0 ). As a form in $\zeta$ it is of degree $(0,0)$ and takes values in $O(-1)$. Clearly $Q$ is not a projective form but we claim that $\bar{\partial} Q$ is. It is enough to prove this in each $\pi^{-1}\left(U_{j}\right)$, so assume e. g. that $z_{0} \neq 0$ and put

$$
\tilde{Q}=\left(Q_{0}-\frac{1}{z_{0}}, Q_{1}, \ldots, Q_{n}\right)
$$


Then the form

$$
\tilde{Q}=\sum_{0}^{n} \tilde{Q}_{j} d z_{j}
$$

is a projective form in $z$ with values in $O(0)$, hence the same holds for $\bar{\partial} \tilde{Q}$, which equals $\bar{\partial} Q$. Note that $\bar{\partial} Q$ also takes values in $O(0)$ as a $(0,0)$-form in $\zeta$. In analogy with formulas (1) and (2) we put

$$
K=\sum_{k=0}^{n-1}(-1)^{k}\left(\begin{array}{c}
N \\
k
\end{array}\right)\langle Q, \zeta\rangle^{N-k} \frac{s \wedge(\bar{\partial} s)^{n-1-k} \wedge(\bar{\partial} Q)^{k}}{\langle s, \zeta\rangle^{n-k}}
$$

$$
P=(-1)^{n+1}\left(\begin{array}{l}
N \\
n
\end{array}\right)\langle Q, \zeta\rangle^{N-n}(\bar{\partial} Q)^{n}
$$

Considered as a form in $z$ the $k$-th term in $K$ takes values in $O(l)$ with

$$
l=-(N-k)+(n-k)=n-N
$$

and as a form in $z$ in $O(-l)$. As it is easy to see the same goes for $P$. We can now state:

Theorem 1. Let $f$ be $a(n, q)$-form in $C^{1}(\bar{D})$ with values in $O(l)$ where $l \leq n$. Put $N=n-l$ and define $K_{q}$ and $P_{q}$ as the components of bidegree $(n, q)$ in $z$ and $(0, n-q-1)$ in $\zeta$ of $K$ and $P$ respectively. Then

$$
\begin{aligned}
& f(z)=C_{n, q}\left\{\int_{\partial D} f(s) \wedge K_{q}(\zeta, z)-\int_{D} \bar{\partial} f(\zeta) \wedge K_{q}(\zeta, z)+\right. \\
& \left.+(-1)^{n+q+1}\left(\bar{\partial} \int_{D} f(\zeta) \wedge K_{q-1}(\zeta, z)+\int_{D} f(\zeta) \wedge P_{q}(s, z)\right)\right\}
\end{aligned}
$$

Proof: The hypothesis quarantees that $f \wedge K_{q}$ etc are scalarvalued as forms in $\zeta$ so the integrals make sense. By a partition of unity we may assume that $f$ has its support in one of the sets $U_{j}$, say in $U_{0}$. We may then also assume $z$ lies in $U_{0}$ since $U_{0}$ is dense in $\mathrm{P}^{n}$. Put

$$
s^{\prime}=\left(s_{1}, \ldots, s_{n}\right)
$$

and define $Q^{\prime}, \zeta^{\prime}, z^{\prime}$ in the same way. Then ii) implies that

$$
s_{0} z_{0}+\left\langle s^{\prime}, z^{\prime}\right\rangle=0
$$

so

$$
\begin{aligned}
& \langle s, \varsigma\rangle=s_{0} \varsigma_{0}+\left\langle s^{\prime}, z^{\prime}\right\rangle=s_{0}\left\langle s^{\prime}, \frac{\varsigma^{\prime}}{\varsigma_{0}}-\frac{z^{\prime}}{z_{0}}\right\rangle= \\
& =\left\langle s^{\prime}\left(\frac{\varsigma^{\prime}}{\varsigma_{0}}, \frac{z^{\prime}}{z_{0}}\right), \frac{\varsigma^{\prime}}{\varsigma_{0}}-\frac{z^{\prime}}{z_{0}}\right\rangle
\end{aligned}
$$


by i). In the same way

$$
\begin{aligned}
& \langle Q, \varsigma\rangle=Q_{0} \varsigma_{0}+\left\langle Q^{\prime}, \varsigma^{\prime}\right\rangle=\varsigma_{0}\left(\frac{1}{z_{0}}+\left\langle Q^{\prime}, \frac{\zeta^{\prime}}{z_{0}}-\frac{z^{\prime}}{z_{0}}\right\rangle\right)= \\
& =\frac{\varsigma_{0}}{z_{0}}\left(1+\left\langle Q^{\prime}\left(\frac{\zeta^{\prime}}{\varsigma_{0}}, \frac{z^{\prime}}{z_{0}}\right), \frac{\varsigma^{\prime}}{\varsigma_{0}}-\frac{z^{\prime}}{z_{0}}\right\rangle\right) .
\end{aligned}
$$

We also have

$$
\sum_{0}^{n} s_{j} d z_{j}=z_{0} \sum_{1}^{n} s_{j} d \frac{z_{j}}{z_{0}}=\frac{z_{0}}{\varsigma_{0}} \sum_{1}^{n} s_{j}\left(\frac{\varsigma^{\prime}}{\varsigma_{0}}, \frac{z^{\prime}}{z_{0}}\right) d \frac{z_{j}}{z_{0}}
$$

and

$$
\begin{aligned}
& \bar{\partial} \sum_{0}^{n} Q_{j} d z_{j}=\bar{\partial}\left(\frac{1}{z_{0}}+z_{0} \sum_{0}^{n} Q_{j} d \frac{z_{j}}{z_{0}}\right)= \\
& =\bar{\partial} \sum_{1}^{n} Q_{j}\left(\frac{\zeta^{\prime}}{\varsigma_{0}}, \frac{z^{\prime}}{z_{0}}\right) d \frac{z_{j}}{z_{0}},
\end{aligned}
$$

Since $\varsigma^{\prime} / s_{0}$ and $z^{\prime} / z_{0}$ are inhomogenous coordinates on $U_{0},(6)$ follows directly from Theorem 0 , applied to the form $s_{0}^{N-n} f$.

So far we have considered only $(n, q)$ forms. The case of $(0, q)$ forms is dual, One way to look at things would be to change the role of the variables. So let us define

$$
\begin{aligned}
& s^{*}(\zeta, z)=s(z, \zeta) \\
& Q^{*}(\zeta, z)=Q(z, \zeta)
\end{aligned}
$$

and $K^{*}, P^{*}$ as

$$
\begin{aligned}
& K^{*}=\sum_{0}^{n-1}(-1)^{k}\left(\begin{array}{l}
N \\
k
\end{array}\right)\left\langle Q^{*}, z\right\rangle^{N-k} \frac{s^{*} \wedge\left(\bar{\partial} s^{*}\right)^{n-1-k} \wedge\left(\bar{\partial} Q^{*}\right)^{k}}{\left\langle s^{*}, z\right\rangle^{n-k}} \\
& P^{*}=(-1)^{n+1}\left(\begin{array}{c}
N \\
n
\end{array}\right)\left\langle Q^{*}, z\right\rangle^{N-n}\left(\bar{\partial} Q^{*}\right)^{n}
\end{aligned}
$$

so that

$$
K^{*}(\varsigma, z)=K(z, \varsigma) \text { and } P^{\cdot}(\varsigma, z)=P(z, \varsigma) .
$$

Then we find, either by repeating the proof of Theorem 1 or just by taking duals of $(6)$,

Theorem 1'. Let $f$ be $a(0, q)$-form in $C^{1}(\vec{D})$ with values in $O(l)$ where $l \geq-n$. Let $N=n+l$. Then

$$
\begin{aligned}
& f(z)=C_{n, q}\left\{\int_{\partial D} f(\zeta) \wedge K_{q}^{*}(\zeta, z)-\int_{D} \bar{\partial} f(\zeta) \wedge K_{q}^{*}(\zeta, z)+\right. \\
& \left.+(-1)^{q+1}\left(\bar{\partial} \int_{D} f(\zeta) \wedge K_{q-1}^{*}(\zeta, z)+\int_{D} f(\zeta) \wedge P_{q}^{*}(\zeta, z)\right)\right\}
\end{aligned}
$$


Remark. Recall that the bundle of $(n, 0)$-forms on $\mathbf{P}^{n}$ is isomorphic to $O(-(n+1))$, so that a $O(l)$-valued $(n, q)$-form can be considered as a $O(l-$ $(n+1))$-valued $(0, q)$-form. Then we see that Theorem 1 gives a Koppelman formula for $(0, q)$-forms with values in $O(l)$ for $l \leq-1$. Hence, together with Theorem I' we have formulas for all the line bundles $O(l)$, and actually both formulas work if $-n \leq l \leq-1$.

We shall now exemplify Theorem 1 by giving a choice of $s$ and $Q$ that works for $D=\mathrm{p}^{n}$. As $Q$ we can take

$$
Q_{j}=\frac{\bar{z}_{j}}{|z|^{2}} \quad \text { so that } \quad Q=\partial \log |z|^{2} .
$$

To chose $s$ we start by deciding what

$$
\langle s, \varsigma\rangle=: \Phi(\zeta, z)
$$

should be. By analogy with the Bochner-Martinelli formula in $\mathbb{C}^{n}$ we take for $\Phi$ the square of the distance between $\zeta$ and $z$.

If $\zeta$ an $z$ are in $\complement^{n+1}$ we put

$$
\widetilde{\Phi}=|\zeta \wedge z|^{2}
$$

where $\zeta \wedge z$ denotes the exterior product and we take norms in $\wedge^{2} \mathbb{C}^{n+1}$. Thus $\tilde{\Phi}(\zeta, z)=0$ precisely when $[\zeta]=[z]$. Then put

$$
\tilde{s}_{j}=\frac{\partial \tilde{\Phi}}{\partial \zeta_{j}}
$$

We claim that

$$
\langle\tilde{s}, s\rangle=\tilde{\Phi} \text { and }\langle\tilde{s}, z\rangle=0
$$

For this, note that for $z$ fixed $\tilde{\boldsymbol{\Phi}}$ is a quadratic form.

$$
\tilde{\Phi}(\zeta, z)=\sum a_{j k}(z) \zeta_{j} \bar{\zeta}_{k} .
$$

The corresponding bilinear form is

$$
\sum a_{j k}(z) w_{j} \bar{\zeta}_{k}=\langle w \wedge z, \zeta \wedge z\rangle
$$

where the scalar product is in $\wedge^{2} c^{n+1}$. Hence

$$
\sum w_{j} \frac{\partial \tilde{\Phi}}{\partial \zeta_{j}}=\langle w \wedge z, \zeta \wedge z\rangle
$$


which equals 0 for $w=z$ and $\tilde{\Phi}$ for $w=\varsigma$. Thus condition ii) is satisfied and to get also i) we replace $\tilde{s}$ by

$$
s=\frac{\tilde{s}}{|\zeta|^{2}|z|^{2}} .
$$

Then

$$
\langle s, s\rangle=\Phi=: \frac{\tilde{\Phi}}{|s|^{2}|z|^{2}}
$$

the natural distance function on $\mathrm{P}^{n}$. Using those choices of $s$ and $Q$ we obtain the following well known corollary to Theorems 1 and I' (cf Theorem 1.4 in [3]).

Corollary. For the Dolbeault cohomology groups of $\boldsymbol{p}^{n}$ we have:

$$
H^{0.9}\left(\mathbf{P}^{n}, O(l)\right)=0 \text { if }
$$

a) $0 \leq q<n$ and $l<0$ or

b) $0<q \leq n$ and $-n \leq l$.

In case $l \geq 0$ we have the following representation formula for an element of $H^{0,0}\left(\mathbf{P}^{n}, O(l)\right)$

$$
f(z)=C_{n} \int_{\mathbf{F} n}\left(\frac{z \cdot \bar{\zeta}}{|\zeta|^{2}}\right)^{l} f(\zeta)\left(\partial \bar{\partial} \log |\zeta|^{2}\right)^{n} .
$$

Proof: If $f$ is $\bar{\partial}$-closed a $(n, q)$-form and $l \leq n$, Theorem 1 gives

$$
f(z)=C_{n}\left\{\bar{\partial} \int_{p n} f(\zeta) \wedge K_{q-1}(\zeta, z)+\int_{p n} f(s) \wedge P_{q}(\zeta, z)\right\} .
$$

But $P_{q}=0$ if $q<n$, so a) follows by identifying $f$ with a $(0, q)$-form with values in $O(l-(n+1))$. In the same way b) follows from (6') since $P^{*}$ is of bidegree $(0,0)$ in $z$. Formula (8) follows from $\left(6^{\prime}\right)$ when $q=0$.

\section{2. $q$-concave domains in $p^{n}$}

Let $D$ be a domain with smooth boundary in $p^{n}$. We say (cf. [3]) that $D$ is $q$-concave if for each point $[z] \in D$ there is a $q$-dimensional projective plane containing $[z]$ that lies entirely within $D$. We assume moreover that the plane can be chosen so that it depends smoothly on $[z]$.

Example: Let

$$
D_{q}=\left\{[z] ;\left|z_{0}\right|^{2}+\ldots,\left|z_{q}\right|^{2}>\left|z_{q+1}\right|^{2}+\ldots,\left|z_{n}\right|^{2}\right\} .
$$


Write

$$
z^{\prime}=\left(z_{0}, \ldots, z_{q}\right), z^{n}=\left(z_{q+1}, \ldots, z_{n}\right) .
$$

We shall describe all the $q$-planes in $D$. Any such plane can be given as

$$
\Pi=\{[z]\} ; A z=0\}
$$

where $A$ is $(n-q) \times(n+1)$ matrix of maximal rank. Since $\Pi \subseteq D$

$$
A z=0 \rightarrow\left|z^{\prime}\right|>\left|z^{\prime \prime}\right|
$$

Write

$$
A=\left(A^{\prime}, A^{\prime \prime}\right)
$$

where $A^{\prime}$ is of order $(n-q) \times(q+1)$ and $A^{\prime \prime}$ of order $(n-q) \times(n-q)$. Then

$$
A z=A^{\prime} z^{t}+A^{\prime \prime} z^{\prime \prime}
$$

From this we see that $A^{n}$ must be nonsingular since otherwise some point $z=\left(0, z^{\prime \prime}\right)$ would lie in $D$ contradicting (1). Hence we may multiply $A$ from the left by $\left(A^{\prime \prime}\right)^{-1}$ which doesn't change the plane $\Pi$, i. e. we may assume $A^{\prime \prime}=I$. Then (1) says that $\left\|A^{\prime}\right\|<1$. In conclusion we see that the set of $q$-planes in $D_{q}$ can be identified with the set of $(n-q) \times(q+1)$ matrices of norm strictly less than 1 .

Let $[s]$ be a point in $D_{q}$ and let $A_{\xi}^{\prime}$ be the matrix corresponding to the linear map

$$
z^{\prime} \rightarrow-\frac{z^{\prime} \cdot \vec{\zeta}}{\left|\zeta^{\prime}\right|^{2}} \zeta^{\prime \prime}
$$

Since $\zeta \in D_{q},\left\|A_{\zeta}^{\prime}\right\|<1$. Moreover $A_{\zeta}^{\prime} \zeta^{\prime}+\zeta^{\prime \prime}=0$ so $[\zeta \mid$ lies in the plane defined by $A_{\zeta}^{t}$. Hence $D_{q}$ is $q$-concave.

We shall now construct a representation formula for $(n, r)$ forms in a $q^{-}$ concave domain.

By hypothesis we can find, in a smoth way, for each $|z| \in D$ a $q$-plane, $\Pi(z)$, such that

$$
[z] \in \Pi(z) \subseteq D
$$

At least locally, $I$ can be represented at the set of $\zeta$ such that

$$
\left\langle\xi^{k}(z), \varsigma\right\rangle=\sum_{0}^{n} \xi_{J}^{k}(z) s_{j}=0, k=1, \ldots,(n-q)
$$

where the functions $\xi^{k}$ are smooth and homogenous of degree 0 . Then we can choose, locally in $z$, functions $\varphi^{1}, \ldots, \varphi^{n-8}$ depending on $\zeta$ and $z$ such that

$$
\sum_{1}^{n-q}\left\langle\varphi^{k}(\zeta, z) \xi^{k}(z), \zeta\right\rangle \geq 0
$$


with equality if and only if $[s] \in \Pi(z)$. One possible choice is

$$
\varphi^{k}=\overline{\left\langle\xi^{k}, \zeta\right\rangle} /|s|^{2}
$$

but we shall see later that the choice is not important. Let us note however that we can also assume that $\varphi^{*}$ is homogenous of degree -1 in $\zeta$ and 0 in $z$. By means of a partition of unity in $z$ we can finally find a function

$$
s^{\prime}: \bar{D}_{\xi} \times D_{z} \rightarrow \boldsymbol{C}^{n+1}
$$

such that

$$
\left\langle s^{\prime}(\zeta, z), \zeta\right\rangle \geq 0 \text { with equality iff }|\zeta| \in \Pi(z)
$$

Moreover $s^{t}$ can be represented as

$$
s^{\prime}=\sum_{1}^{\pi-q} \varphi^{\star}(\zeta, z) \xi^{k}(z)
$$

locally in $z$. Hence, for $|\varsigma| \in \partial D s^{t}$ satisfies the requirements i) - iii) of the previous section. Our next objective is to modify $s$ for $[s \mid \in D$ so that those conditions will be satisfied for all $[\zeta\} \in \bar{D}$.

To this end we let $s^{\prime \prime}$ denote the choice of $s$ of the previous section i. e.

$$
s_{j}^{\prime \prime}=\frac{\partial \tilde{\Phi}}{\partial \zeta_{j}} /|\zeta|^{2}|z|^{2},
$$

and let

$$
s=s^{t}+\rho(\zeta) s^{n}
$$

where $\rho$ is a function that is positive inside $D$ and vanishes to order $I$ on $\partial D$.

Now we can define the kernels $K$ and $P$ by formulas (4) and (5) of section (1). We will leave the choice of $Q$ open for the time being, requiring however that $Q$ be independent of $\zeta$ (and homogenous of degree (-1) in $z$ as always). Recall that this is the case for the "standard choice" $Q=\partial \log |z|^{2}$.

Let us now see what the representation formula in Theorem 1 gives when $r \leq q$ (we are dealing with forms of bidegree $(n, r)$ in a $q$-concave domain). We also suppose $q<n$, since $q=n$ means $D=P^{n}$, which is the case treated in section 1 .

Since the kernel $P$ is of pure bidegree $(n, n)$ in $z$ we have $P_{n, r}=0$. Hence the only obstruction to solvability of the $\bar{\partial}$-equation comes from the boundary integral which we will now evaluate. In our definition of $s,(5)$, the second term 
gives no contribution when $\varsigma \in \partial D$. This is clear if $\rho$ is not differentiated, but it also holds for the differentiated term

$$
\vec{\partial} \rho \wedge s^{\prime \prime},
$$

since $f$ is of full degree in $d \zeta$ so that $\bar{\partial} \rho$ can be replaced by $d \rho$ in the integral, and $d \rho=0$ on $\partial D$.

Hence $s$ can be replaced by $s^{\prime}$ in the calculation of

$$
\int_{\zeta \in \partial D} f(\zeta) \wedge K_{n, r}(\zeta, z)
$$

and we will then use (4). We find

$$
\begin{aligned}
& \bar{\partial} s^{\prime}=\bar{\partial}_{z} s^{t}+\bar{\partial}_{\xi} s^{\prime}= \\
& =\sum_{i}^{n-q} \bar{\partial}_{z} \varphi^{k} \wedge \xi^{k}+\sum_{1}^{n-q} \varphi^{k} \bar{\partial}_{z} \xi^{k}+\sum_{1}^{n-q} \bar{\partial}_{\zeta} \varphi^{k} \xi^{k},
\end{aligned}
$$

where we write $\xi^{k}$ also for the form

$$
\sum_{j=0}^{n} \xi_{j}^{k} d z_{j} .
$$

We are looking for the component of $K$ that is of degree $(n-r-1)$ in $d \bar{\zeta}$ and only the third term in (6) contributes. Hence the part of

$$
s^{\prime} \wedge\left(\bar{\partial} s^{\prime}\right)^{n-k-1}
$$

that we are interested in must contain a factor

$$
\sum \varphi^{k} \xi^{k} \wedge\left(\sum \bar{\partial}_{\xi} \varphi^{k} \wedge \xi^{k}\right)^{n-r-1}
$$

which means that we have a product of $(n-r)$ of the forms $\xi^{k}, k=1, \ldots,(n-q)$. Thus, if $\tau<q$, some form $\xi^{k}$ must occur twice, whence

$$
K_{n, r}=0 \text { for } \varsigma \in \partial D \text { if } r<q .
$$

From this it follows immediately:

Theorem 2. Let $f$ be a $\bar{\partial}$-closed form of bidegree $(n, r)$ in the $q$-concave domain $D$, taking values in the line bundle $O(l)$, where $l \leq n$. Assume $r<q$. Then $f$ is $\bar{\partial}$-exact and we have

$$
f(z)=c_{n}\left\{\vec{\partial}_{z} \int_{D} f(s) \wedge K_{n, r}(s, z)\right\}
$$


See also [3, Theorem 2.2]. In other words

$$
H^{n,+}(D, O(l))=0
$$

if $r<q, l \leq n$. The first bidegree where we have non-vanishing cohomology is $r=q$ which we shall study in the remainder of this paper.

If $r=q,(7)$ equals

$$
a_{n, q} \omega_{q}^{\prime}(\varphi) \wedge \xi^{1} \wedge \ldots, \xi^{n-q}
$$

where

$$
a_{n, q}=(n-q-1) !(-1)^{(n-q+1)(n-q) / 2}
$$

and

$$
\omega_{q}^{\prime}(\varphi)=\sum_{1}^{n-q}(-1)^{m} \varphi^{m} \underset{j \neq m}{\wedge} \bar{\partial}_{s} \varphi^{j}
$$

Our kernel is

$$
K=\sum_{k=0}^{n-1}\left(\begin{array}{l}
N \\
k
\end{array}\right)\langle Q, \zeta\rangle^{N-k}(-1)^{k} \frac{s \wedge(\bar{\partial} s)^{n-k-1} \wedge \bar{\partial} Q}{\langle s, \zeta\rangle^{n-k}}
$$

and we are interested in $K_{n, q}$ when $\varsigma \in \partial D$. Let us use the notation

$$
\begin{aligned}
& \langle\varphi, \xi, \zeta\rangle=\sum \varphi^{m} \xi_{j}^{m} \zeta_{j}=\left\langle s^{\prime}, \zeta\right\rangle \\
& \varphi^{\alpha}=\left(\varphi^{1}\right)^{\alpha_{1}} \ldots,\left(\varphi^{n-q}\right)^{\alpha_{n-q}} \text { if } \alpha=\left(\alpha_{1}, \ldots, \alpha_{n-q}\right) \\
& (\bar{\partial} \xi)^{\alpha}=\left(\bar{\partial} \xi^{1}\right)^{\alpha_{1}} \wedge \ldots,\left(\bar{\partial} \xi^{n-q}\right)^{\alpha_{n-q}} .
\end{aligned}
$$

Since all the forms $\xi^{m}$ occur exactly once in (8), the first term in (6) will not give any contribution when we compute

$$
s \wedge(\bar{\partial} s)^{n-k-1} .
$$

Hence we find for $\varsigma \in \partial D$

$$
\begin{gathered}
K_{n, q}= \\
=a_{n, q} \sum_{\substack{k+|\alpha|=q \\
k \leq N}} b_{N, k, \alpha}\langle Q, \zeta\rangle^{N-k} \frac{\varphi^{\alpha} \omega_{q}^{\prime}(\varphi) \wedge(\bar{\partial} \xi)^{\alpha} \wedge(\bar{\partial} Q)^{k} \wedge \xi^{1} \wedge \ldots, \xi^{n-q}}{\langle\varphi, \xi, \zeta\rangle^{n-k}}
\end{gathered}
$$

where $a_{n, q}$ is some constant (not the same as before) and

$$
b_{N, k, \alpha}=\frac{(n-k-1) !}{\alpha !}\left(\begin{array}{c}
N \\
k
\end{array}\right)(-1)^{k}
$$


Theorem 3. (cf. $[3$, Theorem 2.2]). Let $f$ be a $\bar{\partial}$-closed form of bidegree $(n, q)$ in the $q$-concave domain $D$, taking values in the line bundle $O(l)$, where $l \leq n$. Assume $r<q$. Then $f$ is $\bar{\partial}$-exact if and only if $T(f)=0$ where

$$
T(f)(z)=\int_{\partial D} f(s) \wedge K_{n, q}(\zeta, z)
$$

In that case

$$
f(z)=\bar{\partial}_{z} c_{n, q} \int_{D} f(\zeta) \wedge K_{n, q-1}(\zeta, z)
$$

Proof: It is clear that if $T(f)=0$ then $f$ is $\bar{\partial}$-exact so all we have to prove is that if $f$ is $\bar{\partial}$-exact then $T(f)=0$. Assume

$$
f=\bar{\partial} g \text {. }
$$

Then

$$
\int_{\partial D} f \wedge K_{n, q}=(-1)^{n+q} \int_{\partial D} g \wedge \bar{\partial}_{\zeta} K_{n, q}=(-1)^{n+q} \int_{\partial D} g \wedge d_{\zeta} K_{n, q} .
$$

But the kernels $K$ and $P$ satisfy

$$
d K=P \quad \text { where } \quad \varsigma \neq z
$$

(this is what makes Koppelmans formula true). Considering the components of bidegree $(n, q)$ in $z$ and $(0, n-q-1)$ in $\varsigma$ we get

$$
d_{\zeta} K_{n, q}+d_{z} K_{n, q-1}=0
$$

if we recall $P_{n, r}=0, r<n$. But we have already noted that

$$
K_{n, r}=0 \text { for } s \in \partial D \text { and } r<q \text {. }
$$

Hence

$$
d_{\varsigma} K_{n, q}=0 \text { for } \varsigma \in \partial D
$$

and we are done.

If we put further restrictions on the homogenuity, $l$, the condition $T(f)=0$ can be simplified a lot. We assume that

$$
l \leq n-q
$$

so that

$$
N=n-l \geq q .
$$

Actually an analogous discussion could be carried out for all $N \geq 0$, but the case $N \geq q$ is simpler so we will be content with that case.

Let us look at the term whith $k=q, \alpha=(0, \ldots, 0)$ in $(9)$. The term in question is then

$$
\text { Const }\langle Q, \zeta\rangle^{n-q} \frac{\omega_{q}^{t}(\varphi) \wedge \xi^{1} \wedge \ldots, \xi^{n-q} \wedge(\bar{\partial} Q)^{q}}{\langle\varphi, \xi, \zeta\rangle^{n-q}} .
$$

Remembering that $\xi$ and $Q$ do not depend on $\zeta$ we are led to the following: 
Definition. Let $f$ be $a(n, q)$-form with values in $O(l), l \leq n-q$, and let as before $N=n-l$. Then the Fantappie transform of $f$ is defined by

$$
\mathcal{F}(f)\left(\xi^{\mathrm{I}}, \ldots, \xi^{n-q}, \eta\right)=\int_{\partial D} \frac{f \wedge \omega_{q}^{\prime}(\varphi)}{\langle\varphi, \xi, \zeta\rangle^{n-q}}\langle\eta, \zeta\rangle^{N-q} .
$$

A few comments are in order. First, $N$ is chosen so that the form we integrate takes values in $O(0)$, so the integral makes sense. Second, if $q=n-1$ and $l=0$ (so that $N=n$ )

$$
\mathcal{F}(f)(\xi, \eta)=\int_{\partial D} \frac{f \varphi\langle\eta, \zeta\rangle}{\langle\varphi, \xi, \zeta\rangle}=\int_{\partial D} f \frac{\langle\eta, \zeta\rangle}{\langle\xi, \zeta\rangle} .
$$

This is precisely the Fantappié transform of Gindikin-Henkin [2]. In general $\mathcal{F}(f)$ is defined for all

$$
\eta \in \varepsilon^{n+1}
$$

and for all

$$
\xi=\left(\xi^{1}, \ldots, \xi^{n-q}\right) \in D^{*},
$$

where $D^{*}$ is the set of $\xi$ such that the $q$-plane $\Pi_{\xi}$, defined by the equations

$$
\left\langle\xi^{m}, s\right\rangle=0 \quad m=1, \ldots, n-q,
$$

lies in $D$. This quarantees that $\mathcal{F}(f)(\xi, \eta)$ is well defined and holomorphic in $\xi$ if we make an appropiate choice of $\varphi$. In case $q=n-1$ it is evident that the definition of $\mathcal{F}(f)$ does not depend on the choice of $\varphi$. We shall now prove:

Proposition 4. The definition of $\mathcal{F}(f)$ does not depend on $\varphi$, provided $\bar{\partial} f=$ $=0$.

Proof: Assume we have two choices $\left(\varphi_{0}^{m}\right)$ and $\left(\varphi_{1}^{m}\right) m=1, \ldots, n-q$. Thus $\left(\varphi_{s}^{m}\right)$ are functions of $\xi$ and $\zeta$ such that

$$
\left\langle\varphi_{s}, \xi, \zeta\right\rangle \neq 0 \text { for } \xi \in D^{*}, \zeta \in \partial D, s=0,1 \text {. }
$$

If $\chi$ is a scalarvalued function we have the well known homogenuity property

$$
\omega_{q}^{\prime}(\chi \varphi)=\chi^{n-q} \omega_{q}^{t}(\varphi)
$$

Hence we may assume that

$$
\left\langle\varphi_{s}, \xi, \zeta\right\rangle>0, s=0,1 .
$$

Therefore we can define a homotopy

$$
\varphi_{s}=s \varphi_{I}+(1-s) \varphi_{0} \quad 0 \leq s \leq 1
$$

and (12) still holds for $0 \leq s \leq 1$. We next need the following: 
Lemma 5. Let $F\left(\varphi^{1}, \ldots, \varphi^{n-q}\right)$ be homogemous of degree $-(n-q)$. Then the form

$$
A=F(\varphi) \omega_{q}^{2}(\varphi)
$$

is closed.

Proof of Lemma: By Euler's formula

$$
\sum_{m=1}^{n-q} \varphi^{m} \frac{\partial F}{\partial \varphi^{m}}=-(n-q) F
$$

Hence

$$
d A=\sum \varphi^{m} \frac{\partial F}{\partial \varphi^{m}} d \varphi^{\perp} \wedge \ldots, d \varphi^{n-q}+(n-q) F d \varphi^{1} \wedge \ldots, d \varphi^{n-q}=0 .
$$

To continue the proof of the proposition we put

$$
A=\frac{\omega^{\prime}\left(\varphi_{s}\right)}{\left\langle\varphi_{s}, \xi, \zeta\right\rangle^{n-q}}
$$

and consider $A$ as a form in $\varphi$ and $s$ for $\xi, \zeta$ fixed. Then, if we write

$$
A=A_{s}+A^{\prime}
$$

where $A_{s}$ contains $d s$ and $A^{t}$ does not, we have

$$
d_{\varphi} A_{s}+d_{s} A^{\prime}=0
$$

by Lemma 5 . Let

$$
H=\int_{0}^{1} A_{s} .
$$

Then (13) implies that

$$
d_{\varphi} H=\int_{0}^{1}-d_{s} A^{\prime}=\frac{\omega^{\prime}\left(\varphi_{0}\right)}{\left\langle\varphi_{0}, \xi, \zeta\right\rangle^{n-q}}-\frac{\omega^{\prime}\left(\varphi_{1}\right)}{\left\langle\varphi_{1}, \xi, \zeta\right\rangle^{n-q}} .
$$

This in turn implies

$$
\bar{\partial}_{\zeta} H=\frac{\omega^{t}\left(\varphi_{0}\right)}{\left\langle\varphi_{0}, \xi, \zeta\right\rangle^{n-q}}-\frac{\omega^{t}\left(\varphi_{1}\right)}{\left\langle\varphi_{1}, \xi, \zeta\right\rangle^{n-q}}
$$

which inmediately gives the proposition.

For future reference we now note an invariance property of $\mathcal{F}(f)$. Consider $\xi=\left(\xi^{1}, \ldots, \xi^{n-q}\right)$ as a $(n-q) \times(n+1)$ matrix, $\varphi$ as a row matrix and $\zeta$ as a colum. Let $g$ be a nonsingular $(n-q) \times(n-q)$ matrix. Then $g \xi$ defines the 
same $q$-plane as $\xi$, so let us see how $\mathcal{F}(f)$ changes if we change $\xi$ to $g \xi$. Note that

$$
\langle\varphi, g \xi, \zeta\rangle=\langle\varphi g, \xi, \zeta\rangle
$$

so we can regard the change $\xi$ to $g \xi$ as a change of $\varphi$ to $\varphi g$. Thus

$$
\begin{aligned}
& \mathcal{F}(f)(g \xi, \eta)=\int_{\partial D} \frac{f \wedge \omega^{\prime}(\varphi)}{\langle\varphi g, \xi, \zeta\rangle^{n-q}}\langle\eta, \zeta\rangle^{N-q}= \\
& =\operatorname{detg}^{-1} \int_{\partial D} \frac{f \wedge \omega^{\prime}(\varphi g)}{\langle\varphi g, \xi, \zeta\rangle^{n-q}}\langle\eta, \zeta\rangle^{N-q}= \\
& =(\operatorname{detg})^{-1} \mathcal{F}(f)(\xi, \eta)
\end{aligned}
$$

by Proposition 4 . Here we have used the fact

$$
\omega^{\prime}(\varphi g)=\operatorname{detg} \omega^{\prime}(\varphi)
$$

So we have proved

Proposition 6. If $g \in G L(n-q)$

$$
\mathcal{F}(f)(g \xi, \eta)=(\operatorname{det} g)^{-1} \mathcal{F}(f)(\xi, \eta) .
$$

The reason for the introduction of $\mathcal{f}(f)$ was its relation to the operator $T(f)$. More precisely, the term $\alpha=0$ in the definition of $T(f)$ could be written

$$
\text { const. } \quad \mathcal{F}(f)(\xi, \eta) \xi^{1} \wedge \ldots,\left.\xi^{n-q} \wedge(d \eta)^{q}\right|_{\substack{\xi=\xi(z) \\ \eta=Q(s)}}
$$

We now note that all the terms in the definition of $T(f)$ can be expressed in terms of $\mathcal{F}(f)$. If $\alpha=\left(\alpha_{1}, \ldots, \alpha_{n-q}\right)$ is a muiti-index and $a=\left(a_{0}, \ldots, a_{n}\right)$ is a vector we put

$$
\left(a \cdot \frac{\partial}{\partial \xi}\right)^{\alpha}=\left(a \cdot \frac{\partial}{\partial \xi^{1}}\right)^{\alpha_{1}} \ldots,\left(a \cdot \frac{\partial}{\partial \xi^{n-q}}\right)^{\alpha_{n-q}}
$$

where

$$
a \cdot \frac{\partial}{\partial \xi^{m}}=\sum_{0}^{n} a_{j} \frac{\partial}{\partial \xi_{j}^{m}}
$$

Note that

$$
\left(\alpha \cdot \frac{\partial}{\partial \xi}\right)^{\alpha} \frac{1}{\langle\varphi, \xi, \zeta\rangle^{n-q}}=(-1)^{|\alpha|} \frac{(n-q+|\alpha|-1) !}{(n-q-1) !} \frac{\langle a, \zeta\rangle^{|\alpha|} \varphi^{\alpha}}{\langle\varphi, \xi, \zeta\rangle^{n-q+|\alpha|}}
$$

Thus (9) implies that

$$
K_{n, q}=a_{n, q} \sum_{\substack{k+|\alpha|=q \\ k \leq N}} c_{N, k, \alpha}\left(\langle Q, \zeta\rangle^{N-q}\left(Q \cdot \frac{\partial}{\partial \xi}\right)^{\alpha} \frac{1}{\langle\varphi, \xi, \zeta\rangle^{n-q}} \wedge\right.
$$




$$
\left.\wedge \omega^{t}(\varphi) \wedge(\bar{\partial} \xi)^{\alpha} \wedge \xi^{1} \wedge \ldots, \xi^{n-q} \wedge(\bar{\partial} Q)^{k}\right)
$$

where

$$
c_{N, k, \alpha}=\frac{1}{\alpha !}\left(\begin{array}{l}
N \\
k
\end{array}\right)
$$

This in turn implies that

$$
\begin{gathered}
T(f)= \\
=a_{n, q} \sum_{k+|\alpha|=q} c_{N, k, \alpha}\left(\eta \cdot \frac{\partial}{\partial \xi}\right)^{\alpha} \mathcal{F}(f)(\xi, \eta)(d \xi)^{\alpha} \wedge \xi^{1} \wedge \ldots, \xi^{n-q} \wedge(d \eta)^{k}
\end{gathered}
$$

where $\xi=\xi(z)$ and $\eta=Q(z)$. Notice that it does not matter if we write $d \xi$, $d \eta$ or $\bar{\partial} \xi, \bar{\partial} \eta$ since our form is of full degree in $d z$.

Theorem 7. Let $f$ be a $\bar{\partial}$-closed $(n, q)$-form with values in $O(l), l \leq n-q$. Then $f$ is $\bar{\partial}$-exact if and only if $\bar{f}(f)=0$.

Proof: It follows from (16) and Theorem 3 that if $\mathcal{f}(f)=0$ then $f$ is $\bar{\partial}$-exact. On the other hand if

$$
f=\bar{\partial} g
$$

then $f(f)=0$ since

$$
\bar{\partial} \frac{\omega^{\prime}(\varphi)}{\langle\varphi, \xi, \zeta\rangle^{n-q}}=0
$$

by Lemma 5 .

Hence the Fantappié transform induces a one-to-one map on the cohomology groups $H^{n, q}(D, O(l))$ if $l \leq n-q$.

\section{The Radon transform}

For a $\bar{\partial}$-closed form the Fantappié transform coincides with the Radon transform which we shall now define.

Let $\xi^{1}, \ldots \xi^{n-q}$ be a linearly independent set which define the $q-$ plane $\Pi_{\xi}$. We shall now change the convention of the previous paragraph and identify $\xi^{m}$ with the forms

$$
\xi^{m}=\sum_{0}^{n} \xi_{j}^{m} d \zeta_{j}
$$

Definition 8. Let $f$ be $a(n, q)$-form in $D$ with values in $O(l), l \leq n-q$. Put $N=n-l$. Then the Radon transform of $f$ is defined by

$$
R(f)(\xi, \eta)=\int_{\pi_{\zeta}} \frac{\left.\overline{\xi^{1} \wedge \ldots \xi^{n-q}}\right] f(\zeta)}{\left|\xi^{1} \wedge \ldots \xi^{n-q}\right|^{2}}\langle\eta, \zeta\rangle^{N-q},
$$

for $\xi \in D^{*}, \eta \in \mathbb{C}^{n+1}$.

Note that since $f$ is of bidegree $(n, q)$, we obtain a form of bidegree $(q, q)$ after taking inner products with the form $\overline{\xi^{1} \wedge \ldots \xi^{n-q}}$. Thus integration over the $q$-plane $\Pi_{\xi}$ is a well defined operation. 
Theorem 9. Suppose $f$ is a $\bar{\partial}$-closed form of bidegree $(n, q)$ with values in $O(l), l \leq n-q$. Then

$$
\mathcal{F}(f)=c_{n, Q} R(f) \text {. }
$$

Proof: From the definition of $R(f)$ it is clear that $R(f)$ satisfies the same transformation law under a change of frame as does $\mathcal{f}(f)$. Namely, if $g \in$ $\in G L(n-q)$

$$
R(f)(g \xi, \eta)=(\operatorname{detg})^{-1} R(f)(\xi, \eta)
$$

(cf. Prop. 6). Hence we may assume that $\xi^{1} \ldots \xi^{n-q}$ is orthonormal, and we can chose orthonormal coordinates on $\mathrm{C}^{n+1}$ so that

$$
\begin{array}{ccc}
\xi^{1} & =(0, \ldots, 1,0 \ldots 0) \\
\vdots & \\
\xi^{n-q} & =(0, \ldots 0,1)
\end{array}
$$

are the last $(n-q)$ elements of a dual basis. If we write

$$
s^{\prime}=\left(s_{0}, \ldots \varsigma_{q}\right) \quad s^{\prime \prime}=\left(s_{q}+1, \ldots s_{n}\right)
$$

the plane $\Pi_{\xi}$ is then given by $\zeta^{\prime \prime}=0$. In the definition of $\mathcal{f}(f)$ we now take

$$
\begin{gathered}
\varphi^{m}=\overline{\left\langle\xi^{m}, \zeta\right\rangle}=\bar{\zeta}_{q+m}, m=1, \ldots(n-q) . \\
\mathcal{F}(f)(\xi, \eta)=\int_{\partial D} \frac{\omega_{q}^{\prime}\left(\bar{\zeta}^{n}\right)}{\left|\zeta^{\prime \prime}\right|^{2 n-2 q}} \wedge f(\zeta)\langle\eta, \zeta\rangle^{N-q} .
\end{gathered}
$$

Here the integral is $\bar{\partial}$-closed outside the plane

$$
\Pi_{\xi}=\left\{[s] ; s^{\prime \prime}=0\right\},
$$

so what the theorem amounts to is a calculation of

$$
\bar{\partial} \frac{\omega_{q}^{t}\left(\bar{\zeta}^{\prime \prime}\right)}{\left|s^{n t}\right|^{2 n-2 q}} \wedge f(s)\langle\eta, s\rangle^{N-q}
$$

near $\Pi_{\xi}$ in the sense of currents. This is a purely local question and since the plane $\Pi_{\xi}$ is covered by the open sets

$$
U_{j}=\left\{|\zeta| ; \zeta_{j} \neq 0\right\} \quad, \quad j=0, \ldots q,
$$

we can place ourselves in one of them, say $U_{0}$. We use inhomogenous coordinates $w_{j}=\varsigma_{j} / \varsigma_{0}, j=1, \ldots n$, and let $w^{t}=\left(w_{1} \ldots w_{q}\right), w^{\prime \prime}=\left(w_{q+1}, \ldots w_{n}\right)$. In $U_{0}$

$$
\begin{aligned}
H & =: \frac{\omega_{q}^{\prime}\left(\overline{\zeta^{i \prime}}\right)}{\left|s^{\prime \prime}\right|^{2 n-2 q}} \wedge f(\zeta)\langle\eta, \zeta\rangle^{N-q}= \\
& =\frac{\omega_{q}^{\prime}\left(\bar{w}^{\prime \prime}\right)}{\left|w^{n}\right|^{2 n-2 q}} \wedge\left(w_{0}^{-l} t\right) \wedge\langle\eta,(1, w)\rangle^{N-q}
\end{aligned}
$$


But it is well known that

$$
\bar{\partial} \frac{\omega_{q}^{t}\left(\overline{w^{\prime \prime}}\right)}{\left|w^{\prime \prime}\right|^{2 n-2 q}}=c_{n, q} d \bar{w}_{q+1} \wedge \ldots d \bar{w}_{n} d V
$$

where $d V$ is surface measure on $\Pi_{\xi}$. From this it follows that

$$
\int_{U_{0}} \bar{\partial} H=c_{n, q} \int f^{\prime}\left(w^{\prime}, 0\right)\left\langle\eta,\left(1, w_{0}^{\prime}\right)\right\rangle^{N-q} d V\left(w^{\prime}\right)
$$

where $f^{\prime}$ is the coefficient of

$$
d \bar{w}_{1} \wedge \ldots d \bar{w}_{q} \wedge d w_{1} \wedge \ldots d w_{n}
$$

of the form $w_{0}^{-\ell} f$. But this is precisely

$$
\left.c_{n, q} \int_{\Pi_{\varepsilon}}\left(d \bar{\zeta}_{q+1} \wedge \ldots d \bar{\zeta}_{n}\right)\right] f\langle\eta, \zeta\rangle^{N-q}
$$

so we are done.

Proposition 10. Let $\psi(\xi, \eta)=R(f)(\zeta, \eta)$ be the Radon transform of some $\bar{\partial}$-closed form $f$, with values in $O(l)$. Then

a) $\psi(\cdot, \eta)$ is holomorphic in $\xi \in D^{*}$.

b) $\psi(\xi, \cdot)$ is a homogenous polynomial of degree $n-q-l$.

c) if $g \in G L(n-q)$ $\psi(g \xi, \eta)=(\operatorname{det} g)^{-1} \psi(\xi, \eta)$

d) If $\mu \in \mathbb{C}^{n+1}$ lies in the linear span of $\xi^{1}, \ldots \xi^{n-q}$, then $\psi(\xi, \eta+\mu)=$ $=\psi(\xi, \eta)$.

Proof: a) follows from Theorem 9, b) and c) are obvious from the definition, as is $d)$ since $\langle\mu, \zeta\rangle=0$ on $\Pi_{\xi}$ if $\mu$ lies in the span of $\xi^{1}, \ldots \xi^{n-q}$.

Let $\psi$ be a function that satisfies a) - d). Property c) leads us to introduce the form

$$
\Omega_{\psi}=\psi(\xi, \eta) \xi^{1} \wedge \ldots \xi^{n-q}
$$

where from now on we have returned to the old convention of letting

$$
\xi^{m}=\sum_{0}^{n} \xi_{j}^{m} d z_{j}
$$

We consider $\Omega_{\psi}$ as being defined on the bundle over $D$

$$
\begin{aligned}
& E=\left\{(([z], \eta), \xi) ;\left.([z], \eta) \in O(-1) \otimes C^{n+1}\right|_{D}, \xi \in D^{*},\right. \\
& \left.\langle\eta, z\rangle=1 \text { and }\left\langle\xi^{m}, z\right\rangle=0, m=1, \ldots n-g\right\} .
\end{aligned}
$$


Then $\Omega_{\psi}$ takes values in $O(l)$. Observe that the condition $\left\langle\xi^{m}, z\right\rangle=0$ guarantees that $\Omega_{\psi}$ is projective, and that a section, $t$, to $E$ is a map

$$
[z] \stackrel{t}{\rightarrow}([z], \eta(z), \xi(z))
$$

where $\eta(z)$ is homogenous of degree -1 and $\xi(z)$ is homogenous of degree 0 . Note also that if $g \in G L(n-q)$ and we define

$$
\tilde{g}: E \rightarrow E
$$

by $\tilde{g}([z], \eta, \xi)=(|z|, \eta, g \xi)$, then $\tilde{g}^{*}\left(\Omega_{\psi}\right)=\Omega_{\psi}$, by c). In particular the pullback of $\Omega_{\psi}$ under any section $t$, depends only on the plane $\Pi_{\xi}$ defined by $\xi$ and not on the choice of frame.

Inspired by formula (16) of section 2 we consider together with $\Omega_{\psi}$ the form

$$
\hat{\Omega}_{\psi}=\sum_{\substack{k+|\alpha|=q \\ k \leq N}} c_{N, \boldsymbol{k}, \alpha}\left(\eta \cdot \frac{\partial}{\partial \xi}\right)^{\alpha} \psi(\xi, \eta)(d \xi)^{\alpha} \wedge \xi^{1} \wedge \ldots \xi^{n-q} \wedge(d \eta)^{k}
$$

where still

$$
c_{N, k, \alpha}=\frac{1}{\alpha !}\left(\begin{array}{c}
N \\
k
\end{array}\right)
$$

It is not hard to check that $\hat{\Omega}_{\psi}$ also satisfies the invariance property

$$
\tilde{g}^{*}\left(\hat{\Omega}_{\psi}\right)=\hat{\Omega}_{\psi}
$$

if $g$ is a matrix in $G L(n-q)$. As a matter of fact this even holds if $g$ depends on $z$, since each differentiation of $g$ produces a factor $\xi^{m}$ and we already have a complete set of those forms.

From this it follows that if

$$
t: D \rightarrow E
$$

is a section to $E$ the pullback

$$
t^{*}\left(\hat{\Omega}_{\psi}\right)
$$

only depends on the $q$-plane defined by $\xi(z)$, and not on the choice of frame. In particular, if $\left(t_{s}\right)$ is a set of local sections to $E$ such that the covectors $\xi_{s}$ define the same $q$-planes on overlaps we have that

$$
t_{s}^{*}\left(\hat{\Omega}_{\psi}\right)
$$

is a global form. In the sequel we will not distinguish between two sections if they define the same $q$-planes.

From the previous section it is clear that if $\psi=R(f)$, where $f$ is some $\bar{\partial}$-closed form, and if $t$ is some section to $E$ then

$$
\bar{\partial} t^{*}\left(\hat{\Omega}_{\psi}\right)=0
$$

Thus, in addition to the properties a) - d) of Proposition 10, $\psi$ has to satisfy a certain differential equation. 
Proposition 11. Let $\psi$ be a function satisfying properties a) - d) of Proposition 10. Then the following conditions are equivalent:

$\left.e_{1}\right)$ If $t$ is any section to $E$ then $\bar{\partial} t^{*}\left(\hat{\Omega}_{\psi}\right)=0$.

$\left.e_{2}\right)$ On $E, d \hat{\Omega}_{\psi}=0$.

$\left.e_{3}\right) \psi$ satisfies the equations

$$
\begin{gathered}
\frac{\partial^{2} \psi}{\partial \xi_{j}^{m} \partial \xi_{i}^{k}}=\frac{\partial^{2} \psi}{\partial \xi_{i}^{m} \partial \xi_{j}^{k}}, \frac{\partial^{2} \psi}{\partial \xi_{j}^{m} \partial \eta_{i}}=\frac{\partial^{2} \psi}{\partial \xi_{i}^{m} \partial \eta_{j}} \\
m, k=1, \ldots n-q, j=0, \ldots n .
\end{gathered}
$$

(In case $l=n-q, \psi$ is independent of $\eta$ so the second set of equations is void. If moreover $q=n-1$ the first set of equations is also void.)

It is clear that $e_{2}$ implies $e_{1}$, since

$$
\bar{\partial} t^{*}\left(\hat{\Omega}_{\psi}\right)=d t^{*}\left(\hat{\Omega}_{\psi}\right)=t^{*}\left(d \hat{\Omega}_{\psi}\right)
$$

Moreover it will follow from the inversion formula that we shall now prove that $e_{1}$ implies $e_{3}$, since it is obvious from the definition of $\mathcal{F}$ that any $\psi$ that can be written

$$
\psi=\mathcal{F}(f)
$$

will satisfy $e_{3}$. What remains to be proved is thus that $e_{3}$ implies $e_{2}$. Since we have found no slick proof of this fact we have resorted to the method of brute computation, some indications of which will be given after the inversion theorem.

In the proof of the inversion formula we assume that $D$ satisfies one extra assumption.

(A). Let

$$
\varsigma \rightarrow \Pi^{j} \quad j=1,2
$$

be two smooth functions that associates to 5 a q-plane $\Pi$ that lies in $D$ and contains 5. Assume $\Pi^{1}$ is defined in a neighbourhood of $\partial D$ and $\Pi^{2}$ in a neighbourhood of a given $q$-plane $\Pi_{0}$ in $D$. Then there is a similar function

$$
\varsigma \rightarrow \Pi \quad s \in \bar{D}
$$

such that $\Pi=\Pi^{1}$ near $\partial D$ and $\Pi=\Pi^{2}$ near $\Pi_{0}$.

Clearly assumption $(A)$ holds if the set of all planes passing through a given point is contractible, which is the assumption in [2]. (Hence in particular it holds for $D=D_{Q}$ see example at the beginning of section 2). In [3] the inversion theorem is stated without extra assumption but no proof is given. 
Theorem 12. For a function $\psi(\xi, \eta)$ to be reprentable as $\psi=R(f)$ with $f \bar{\partial}$-closed it is necessary and sufficient that $\psi$ satisfy properties a) $-d)$ of Proposition 10 and property $e_{1}$ of Proposition 11. In that case

$$
\psi=c_{n, q} R(f) \text { with } f=t^{*}\left(\hat{\Omega}_{\psi}\right)
$$

if $t$ is any section

$$
t: D \rightarrow E
$$

Proof: All that remains to prove is that if $\psi$ satisfies conditions a) $-\mathrm{d}$ ), $e_{1}$ ) then

$$
\psi=c_{n, q} R(f) \text { with } f=t^{*}\left(\hat{\Omega}_{\psi}\right) .
$$

So let $t$ be a section to $E$ and $f x$ a $q$-plane $\Pi_{(0)}$ in $D$. Say $\Pi_{(0)}$ is given by the equations

$$
\left\langle\mu^{1}, \zeta\right\rangle=\ldots\left\langle\mu^{n-q}, \zeta\right\rangle=0
$$

where $\left(\mu^{1}, \ldots \mu^{n-q}\right)$ is orthonormal. We may even choose coordinates so that

$$
\left\langle\mu^{m}, \zeta\right\rangle=\varsigma^{q+m} \quad m=1, \ldots n-q,
$$

since the construction of $\hat{\Omega}_{\psi}$ is invariant if we make dual changes of coordinates in $\zeta, z$ and $\xi, \eta$.

By assumption $e_{1}, \bar{\partial} f=0$, hence

$$
\xi(f)=c_{n, q} R(f),
$$

by theorem 9. Thus the value of $R(f)$ at $\mu$ depends only on the choice of $t$ near $\partial D$, so by assumption $(A)$ we can chose $t$ any way we like near $\Pi_{(0)}$. As $\eta=\eta(s)$ we take as usual

$$
\eta_{j}(\zeta)=\frac{\overline{\zeta_{j}}}{|\zeta|^{2}} \quad z \in \bar{D}
$$

and as $\xi=\xi(s)$ we will take

$$
\xi(\varsigma)=\mu \quad \text { when } \quad s \in \Pi_{(0)}=\Pi_{\mu},
$$

and then extend this definition of $\xi$ to a neighbourhood of $\Pi_{\mu}$. This we do as follows:

For $\zeta$ lying in a neighbourhood of $\Pi_{\mu}$ we denote

$$
\Pi^{s}=\left\{[z]=\left[z^{\prime}, z^{\prime \prime}\right] ; z^{\prime \prime}=\frac{z^{\prime} \cdot \bar{\zeta}^{\prime}}{\left|\zeta^{\prime}\right|^{2}} \varsigma^{\prime \prime}\right\}
$$

where $z^{\prime}=\left(z_{0} \ldots z_{q}\right), z^{\prime \prime}=\left(z_{q+1}, \ldots z_{n}\right)$. Clearly

$$
\left.\Pi^{\zeta}=\Pi_{\mu} \quad \text { if } \quad \zeta \in \Pi_{\mu} \quad \text { (i. e. if } \quad \zeta^{\prime \prime}=0\right),
$$


so $\Pi^{\zeta} \subset D$ if $\zeta$ is close to $\Pi_{\mu}$. As our frame $\xi(\zeta)$ that defines the $q$-plane $\Pi^{\zeta}$ we take $\xi^{m}$ so that

$$
\left\langle\xi^{m}, z\right\rangle=z^{q+m}-\frac{z^{t} \cdot \vec{\zeta}^{\prime}}{\left|\zeta^{\prime}\right|^{2}} \zeta^{q+m}
$$

Hence we get for the forms

$$
\begin{aligned}
& \xi^{m}=\sum_{0}^{n} \xi_{j}^{m}(\zeta) d \zeta_{j}=\sum_{0}^{n} \frac{\partial}{\partial z_{j}}\left\langle\xi^{n}(\zeta), z\right\rangle d \zeta_{j}= \\
& =d \zeta_{q+m}-\zeta_{q+m} \sum_{0}^{q} \frac{\bar{\zeta}_{j}}{\left|\zeta^{\ddagger}\right|^{2}} d \zeta_{j}=d \zeta_{q+m}-\zeta_{q+m} \partial \log \left|\zeta^{\prime}\right|^{2},
\end{aligned}
$$

and

$$
\bar{\partial} \xi^{m}=\varsigma_{q+m} \partial \bar{\partial} \log \left|\zeta^{\prime}\right|^{2}
$$

By definition

$$
R(f)\{\mu, \delta)=\int_{\Pi_{\mu}} \frac{\overline{\mu^{1} \wedge \ldots \mu^{n-q}}}{\left|\mu^{1} \wedge \ldots \mu^{n-q}\right|^{2}} \mid f\langle\delta, \zeta\rangle^{N-q}
$$

where

$$
f=\sum c_{N, k, \alpha}\left(\eta \cdot \frac{\partial}{\partial \xi}\right)^{\alpha} \psi \quad(\bar{\partial} \xi)^{\alpha} \wedge(\bar{\partial} \eta)^{k} \wedge \xi^{1} \wedge \ldots \xi^{n-q} .
$$

Fortunately enough, when $\zeta \in \Pi_{\mu}, \varsigma_{q+m}=0$ for $m=1, \ldots n-q$, so the only term in $f$ that does not vanish on $\Pi_{\mu}$ is the one with $\alpha=0$. When $\zeta \in \Pi_{\mu}$

$$
f=c_{N, q, 0} \psi(\xi, \eta)\left(\bar{\partial} \partial \log \left|\zeta^{\prime}\right|^{2}\right)^{q} \wedge d \delta_{q+1} \wedge \ldots d_{\zeta_{q+m^{\prime}}}
$$

and $\xi(\zeta)=\mu$. Therefore

$$
\left.\frac{\overline{\mu^{1} \wedge \ldots \mu^{n-q}}}{\left|\mu^{1} \wedge \ldots \mu^{n-q}\right|^{2}}\right\} f=c_{N, q, 0} \psi\left(\mu, \frac{\overrightarrow{s^{\prime}}}{\left|\zeta^{t}\right|^{2}}\right)\left(\bar{\partial} \partial \log \left|\zeta^{\prime}\right|^{2}\right)^{q},
$$

and

$$
R(\delta)(\mu, \delta)=c_{N, q, 0} \int_{\Pi_{\epsilon}} \psi\left(\mu, \frac{\bar{\zeta}^{t}}{\left|\zeta^{t}\right|^{2}}\right)\left\langle\delta^{\prime}, \zeta^{\prime}\right\rangle^{N-q}\left(\bar{\partial} \partial \log \left|s^{\prime}\right|^{2}\right)^{q} .
$$

Recalling that $\psi$ is a homogenous polynomial of degree $n-l-q=N-q$ in the variable $\eta$ we find

$$
R(f)(\mu, \delta)=c_{n, q} \psi\left(\mu, \delta^{\prime}\right)
$$

(this is e.g. a consequence of (8) sec. 1). But now property d) gives that

$$
\psi\left(\mu, \delta^{\prime}\right)=\psi(\mu, \delta)
$$

so the proof is complete. 
If $\eta_{j}(s)=\frac{\bar{s}_{i}}{|j|^{2}}$ the inversion formula we obtain is close to the one in $[3]$. But of course we are free to chose $\eta$ in many other ways. If $q=n-1$ we can take

$$
\eta(s)=\frac{\eta_{0}}{\left\langle\eta_{0}, s\right\rangle}
$$

when $s$ lies close to $\partial D$, if $\eta_{0} \in D^{*}$. Then

$$
\bar{\partial} \eta=0
$$

so this time the only non-zero term in $\hat{\Omega}_{\psi}$ is

$$
\left(\eta \cdot \frac{\partial}{\partial \xi}\right)^{n-1} \psi \xi \wedge(d \xi)^{n-1} .
$$

If we use the Fantappie transform instead of the Radon transform our inversion formula can be written

$$
\psi(\mu, \delta)=\left.c \int_{\partial D}\left(\eta \cdot \frac{\partial}{\partial \xi}\right)^{n-1} \psi \xi \wedge(d \xi)^{n-1}\right|_{\xi=\xi(\zeta)} \frac{\langle\delta, \zeta\rangle}{\langle\mu, \zeta\rangle}^{N-(n-1)} .
$$

This formula is the starting point of [2].

Sketch of proof of Proposition 11: As already remarked $e_{2}$ implies $e_{1}$ directly. Moreover since we only used property $e_{I}$ in the proof of Theorem 12, we have that $e_{1}$ implies that

$$
\psi(\xi, \eta)=c \int_{\partial D} \frac{f \wedge \omega_{q}^{\prime}(\varphi)\langle\eta, \zeta\rangle^{N-q}}{\langle\varphi, \xi, \zeta\rangle^{n-q}}
$$

for some $f$. But then $e_{3}$ follows directly by differentiation under the integral sign. Thus all we need to prove is that $e_{3}$ implies $e_{2}$. Here is an indication of one way to see this. Let

$$
\psi^{\alpha}=\left(\eta \cdot \frac{\partial}{\partial \xi}\right)^{\alpha} \psi
$$

Then

$$
\begin{aligned}
d \hat{\Omega}_{\psi} & =\sum_{k, \alpha} c_{N, k, \alpha} d_{\eta} \psi^{\alpha} \wedge(d \xi)^{\alpha} \wedge \xi^{1} \wedge \ldots \xi^{n-q} \wedge(d \eta)^{k}+ \\
& +\sum_{k, \alpha, m} c_{N, k, \alpha} d_{\xi_{m}} \psi^{\alpha} \wedge(d \xi)^{\alpha} \wedge \xi^{1} \wedge \ldots \xi^{n-q} \wedge(d \eta)^{k}+ \\
& +\sum_{k, \alpha} c_{N, k, \alpha}(-1)^{m} \psi^{\alpha}(d \xi)^{\alpha+1_{m}} \wedge \xi^{1} \wedge \ldots \hat{\xi}^{m} \wedge \ldots \xi^{n} \wedge(d \eta)^{k}
\end{aligned}
$$

where we use the notation

$$
\alpha+1_{m}=\left(\alpha_{1}, \ldots \alpha_{m}+1, \ldots \alpha_{n}\right) .
$$


Let $L^{\alpha}$ denote the vector field

$$
L^{\alpha}=\sum \frac{\partial}{\partial \eta_{j}} \psi^{\alpha} \frac{\partial}{\partial z_{j}}
$$

Then

$$
\text { i) } \left.\quad L^{\alpha}\right\rfloor d \eta=-d_{\eta} \psi^{\alpha} \text {. }
$$

If $|\alpha|+k=(q+1)$

$$
(d \xi)^{\alpha} \wedge \xi^{1} \wedge \ldots \xi^{n-q} \wedge(d \eta)^{k}=0
$$

since a projective form can have at most degree $n$ in $d z$. Hence

$$
\begin{aligned}
0 & \left.=L^{\alpha}\right\rfloor(d \xi)^{\alpha} \wedge \xi^{1} \wedge \ldots \xi^{n-q} \wedge(d \eta)^{k}= \\
& \left.=\sum_{m} \alpha_{m}\left(L^{\alpha}\right\rfloor d \xi^{m}\right)(d \xi)^{\alpha-1 m} \wedge \xi^{1} \wedge \ldots \xi^{n-q} \wedge(d \eta)^{k}+ \\
& \left.+\sum_{m}(-1)^{m}\left(L^{\alpha}\right\rfloor \xi^{m}\right)(d \xi)^{\alpha} \wedge \xi^{1} \wedge \ldots \hat{\xi}^{m} \wedge \xi^{n-q} \wedge(d \eta)^{k}+ \\
& \left.+k\left(L^{\alpha}\right\rfloor d \eta\right)(d \xi)^{\alpha} \wedge \xi^{1} \wedge \ldots \xi^{n-q} \wedge(d \eta)^{k-1}
\end{aligned}
$$

Using $e_{3}$ and homogenuity properties of $\psi^{\alpha}$ we find

ii)

$$
\left.L^{\alpha}\right\rfloor d \xi^{m}=(k-N-1) d_{\xi^{m}} \psi^{\alpha-1_{m}}(|\alpha|+k=q+1) .
$$

Using the homogenuity of $\psi$ and the equations

$$
\begin{aligned}
& \sum \xi_{j}^{k} \frac{\partial \psi}{\partial \xi_{j}^{m}}=0 \quad k \neq m \\
& \sum \xi_{j}^{m} \frac{\partial \psi}{\partial \eta_{j}}=0
\end{aligned}
$$

which follow from c) and d) of Proposition 10 respectively, we find

$$
\text { iii) } \left.\quad L^{\alpha}\right\rfloor \xi^{m}=(k-N-1) \alpha_{m} \psi^{\alpha-1_{m}} \cdot(|\alpha|+k=q+1) \text {. }
$$

Inserting i), ii) and iii) in (6), and using the obtained relation in (5) we get

$$
d \hat{\Omega}_{\downarrow}=0 .
$$




\section{Formulas for $(p, q)$ forms}

To start with, let $M$ be a complex manifold and let $E$ be a holomorphic vector bundle over $M$. We suppose $E$ is endowed with some hermitian metric and let $\nabla$ be the unique holomorphic connection in $E$ compatible with the metric. Together with $E$ we consider the dual bundle, $E^{*}$, and denote the pairing between $E^{*}$ and $E$ by

$$
E_{z}^{*} \times E_{z} \ni(\xi, \eta) \rightarrow\langle\xi, \eta\rangle_{z} \in \mathcal{C} .
$$

The connection $\nabla$ induces a dual connection in $E^{*}$ (also denoted $\nabla$ ), by

$$
d\langle\xi, \eta\rangle=\langle\nabla \xi, \eta\rangle+\langle\xi, \nabla \eta\rangle
$$

if $\xi$ and $\eta$ are sections to $E^{*}$ and $E$ respectively. The curvature of $\nabla$ is defined by

$$
\Theta_{E} \eta=\nabla^{2} \eta
$$

The fact that $\nabla$ is holomorphic means that

$$
\nabla=\bar{\partial}+\nabla^{\prime}
$$

where $\nabla^{\prime}$ is an operator of bidegree $(1,0)$. Since $\nabla$ is the canonical connection of a hermitian metric the fact that $\bar{\partial}^{2}=0$ implies that

$$
\left(\nabla^{\prime}\right)^{2}=0
$$

Hence

$$
\Theta_{E} \eta=\left(\nabla^{\prime} \bar{\partial}+\bar{\partial} \nabla^{\prime}\right) \eta
$$

If, with respect to some holomorphic frame

$$
\nabla^{\prime}=\partial+\theta
$$

then

$$
\bar{\partial}\left(\nabla^{\prime} \eta\right)=\bar{\partial} \partial \eta+(\bar{\partial} \theta) \eta-\theta \wedge \bar{\partial} \eta=-\nabla^{\prime}(\bar{\partial} \eta)+(\bar{\partial} \theta) \eta
$$

so that

$$
\Theta_{E} \eta=(\bar{\partial} \theta) \eta
$$

Thus $\Theta_{E}$ operates on a section as multiplication with a matrix of $(1,1)$ forms. We now make the very restrictive assumption that this matrix is a multiple of the identity matrix, i. e. that

$$
\Theta_{E} \eta=\eta \Theta
$$

where $\Theta$ is a (scalarvalued) $(1,1)$ form and multiplication is componentwise. 
Example: Let $L$ be a hermitian line bundle and let

$$
E=L \otimes\left(\mathbb{C}^{N} \times M\right)
$$

If the metric on $L$ is given by $h>0$ in some holomorphic frame then

$$
\theta=(\partial \log h) I
$$

and

$$
\Theta=(\bar{\partial} \partial \log h) I .
$$

In this very special setting we shall now generalize the construction in $[4]$, following the pattern in [1].

Let $\xi$ be an arbitrary section to $E^{*}$ and let $\eta$ be a holomorphic section to $E$. Consider the form

$$
A=e^{\langle\xi, \eta\rangle}\left\langle\bar{\partial} \xi, \nabla^{\prime} \eta\right\rangle^{n}
$$

Since $\eta$ is holomorphic

$$
\bar{\partial} A=e^{\langle\xi, \eta\rangle}\left(\langle\bar{\partial} \xi, \eta\rangle \wedge\left\langle\bar{\partial} \xi, \nabla^{\prime} \eta\right\rangle^{n}-n\left\langle\bar{\partial} \xi, \bar{\partial} \nabla^{\prime} \eta\right\rangle \wedge\left\langle\bar{\partial} \xi, \nabla^{\prime} \eta\right\rangle^{n-1}\right) .
$$

But

$$
\bar{\partial} \nabla^{\prime} \eta=\Theta_{E} \eta=\eta \Theta
$$

since $\bar{\partial} \eta=0$. Hence

$$
\bar{\partial} A=e^{\langle\xi, \eta)}\left\{\langle\bar{\partial} \xi, \eta\rangle \wedge\left\langle\bar{\partial} \xi, \nabla^{\prime} \eta\right\rangle^{n}-n\langle\bar{\partial} \xi, \eta\rangle \wedge\left\langle\bar{\partial} \xi, \nabla^{\prime} \eta\right\rangle^{n-1} \wedge \Theta\right) .
$$

This leads us to define

$$
\begin{aligned}
& A_{j}=e^{\langle\xi, \eta\rangle}\left\langle\bar{\partial} \xi, \nabla^{\prime} \eta\right\rangle^{n-j} \wedge \Theta^{j} \quad \text { and } \\
& B_{j}=\langle\bar{\partial} \xi, \eta\rangle \wedge A_{j} \quad j=0,1, \ldots, n
\end{aligned}
$$

Using the fact that $\bar{\partial} \Theta=0$ we find that

$$
\bar{\partial} A_{j}=B_{j}-(n-j) B_{j+1} \quad \text { (where } \cdot B_{n+1}=: 0 \text { ), }
$$

by repeating the same computation as above. Next we put

$$
A^{*}=: \sum_{0}^{n} \frac{n !}{(n-k) !} A_{k}
$$

and obtain

$$
\bar{\partial} A^{*}=\sum_{0}^{n} \frac{n !}{(n-k) !}\left(B_{k}-(n-k) B_{k+1}\right)=B_{0} .
$$

Note that the form $B_{0}$ contains $(n+1)$ factors $\bar{\partial} \xi$. From this we immediately get 
Lemma 13. Suppose $\bar{\partial} \xi$ takes values in some holomorphic subbundle, $F$, of $E^{*}$ where $\operatorname{rank}(F) \leq n$. Then $\bar{\partial} A^{*}=0$.

Now we let $s$ be a global section of $E^{*}$ and replace $\xi$ in the definition of $A^{*}$ by

$$
\xi=-\bar{\lambda} s, \quad \lambda \in \mathbb{C} .
$$

Then we get a form, still denoted $A^{*}$, defined on $M \times C$, and we have

$$
\left(\bar{\partial}_{\lambda}+\bar{\partial}_{M}\right) A^{*}=0
$$

provided $s$ takes values in some subbundle of $E^{*}$ of rank not exceeding $n$. Write

$$
A^{*}=A_{1}^{*}+A_{\lambda}^{*}
$$

where $A_{\dot{\lambda}}^{*}$ is the component of $A^{*}$ that contains $d \bar{\lambda}$. Then

$$
d_{\lambda} A_{\mathrm{I}}^{*}+\bar{\partial}_{M} A_{\lambda}^{*}=0 .
$$

We now choose a global holomorphic section, $\eta$, to $E$, and suppose that

$$
\operatorname{Re}\langle s, \eta\rangle \geq 0 \text {. }
$$

We can then define

$$
K=\int_{\lambda \in(0, \infty)} A_{\lambda}^{*}
$$

$K$ is a form of degree $(2 n-1)$ defined on the domain in $M$ where strict inequality holds in (1). When we compute the integral we put $d \bar{\lambda}$ to the far left in the form. Then

$$
\bar{\partial} K=-\int_{\lambda \in(0, \infty)} \bar{\partial} A_{\lambda}^{*}=+\int_{\lambda \in(0, \infty)} d_{\lambda} A_{\lambda}^{*}=-\left.A_{i}^{*}\right|_{\lambda=0}=-\Theta^{n} .
$$

We can compute $K$ explicity as follows

$$
K=\sum_{0}^{n-1} \frac{n !}{(n-k) !} K^{k}
$$

where

$$
K^{k}=(n-k)(-1)^{n-k} \int_{0}^{\infty} e^{-\lambda\langle s, \eta)}(\lambda)^{n-k-1} d \lambda\left\langle s, \nabla^{\prime} \eta\right\rangle \wedge\left\langle\bar{\partial} s, \nabla^{\prime} \eta\right\rangle^{n-k-1} \wedge \Theta^{k} .
$$

Hence

$$
K=\sum_{0}^{n-1}(-1)^{n-k} n ! \frac{\left\langle s, \nabla^{\prime} \eta\right\rangle \wedge\left\langle\bar{\partial} s, \nabla^{\prime} \eta\right\rangle^{n-k-1} \wedge \Theta^{k}}{\langle s, \eta\rangle^{n-k}}
$$


This is clearly a generalization of the classical Cauchy-Leray form (take $\nabla^{\prime}=$ $\partial, \Theta=0)$.

The idea to introduce a connection in the Cauchy-Leray form comes from Demailly and Laurent-Thiebaut [4]. However, they consider only the first term $(k=0)$ in the expansion of $K$. In this case

$$
\bar{\partial} K^{0}=0\left\{|\langle s, \eta\rangle|^{-(n-1)}\right)
$$

so that, roughly speaking, $\bar{\partial} K^{0}=0$ modulo lower order terms. This is less precise than our formula but on the other hand holds for arbitrary connections.

Following the same method as in [1] we can now construct weighted formulas. Then we let $Q$ be another global section to $E^{*}$. We assume there is a subbundle, $F$, of $E^{*}$ with rank $(F) \leq n$, such that both $s$ and $\bar{\partial} Q$ take values in $F$. Then we can replace $\xi$ in the definition of $A^{*}$ by

$$
\xi=-\bar{\lambda} s+Q
$$

and define $K$ in the same way as before. We then get by the same computation

$$
\bar{\partial} K=-\left.A_{1}^{*}\right|_{\lambda=0}=-\sum_{0}^{n} \frac{n !}{(n-k) !} e^{\langle Q, n\rangle}\left\langle\bar{\partial} Q, \nabla^{\prime} \eta\right\rangle^{n-k} \wedge \Theta^{k}=P .
$$

Finally, arguing as in $\{1\}$ we can replace the exponential in our kernels $K$ and $P$ by any holomorphic function $G(t)$, such that $G(0)=1$. We then obtain the following two kemels:

$$
\text { (2) } \begin{gathered}
K=\sum_{|\alpha|=n-1} C_{\alpha} G^{\left(\alpha_{2}\right)}(\langle Q, \eta\rangle) \frac{\left\langle s, \nabla^{\prime} \eta\right\rangle \wedge\left\langle\bar{\partial} s, \nabla^{\prime} \eta\right\rangle^{\alpha_{1}} \wedge\left\langle\bar{\partial} Q, \nabla^{\prime} \eta\right\rangle^{\alpha_{2}} \wedge \Theta^{\alpha_{3}}}{\langle s, \eta\rangle^{\alpha_{1}+1}} \\
P=\sum_{|\beta|=n} D_{\beta} G^{\left(\beta_{1}\right)}\{\langle Q, \eta\rangle\rangle\left\langle\bar{\partial} Q, \nabla^{\prime} \eta\right\rangle^{\beta_{1}} \wedge \Theta^{\beta_{2}},
\end{gathered}
$$

where $C_{\alpha}=-(-1)^{\alpha_{1}} n ! /\left(\alpha_{1}+\alpha_{2}+1\right) \alpha !$ and $D_{\beta}=-n ! / \beta_{1} !$

We collect our result so far in:

Proposition 14. Suppose $s$ and $Q$ are global sections to $E^{*}$ and that $\eta$ is a holomorphic global section to $E$. Assume there is a holomorphic subbundle, $F$, of $E^{*}$, with rank $(F) \leq n$, such that $s$ and $\bar{\partial} Q$ take values in $F$. Then, if $K$ and $P$ are defined as in (2)

$$
\vec{\partial} K=P
$$

in $M^{\prime}=\{(s, \eta) \neq 0\}$.

(Up to now we have assumed that

$$
\operatorname{Re}\langle s, \eta\rangle \geq 0,
$$


but since it follows directly from the formula for $K$ that $K$ is unchanged if we multiply $s$ by a scalar function, we may drop this assumption).

We are now ready to apply this construction to projective space.

As $M$ we take

$$
M=\mathbb{C}_{\zeta}^{n+1} \backslash\{0\} \times \mathbb{C}_{z}^{n+1} \backslash\{0\},
$$

and as our vectorbundle $E$ we take

$$
M \times \mathbb{C}^{n+1} \text { so that also } E^{*}=M \times \mathbb{C}^{n+1} .
$$

Let

$$
F=\left\{(\zeta, z, \xi) ;(\zeta, z) \in M \text { and } \sum_{0}^{n} z_{j} \xi_{j}=0\right\},
$$

and consider $F$ as a subbundle of $E^{*}$ (note rank $F=n$ ). If $\eta=\left(\eta_{0}, \ldots, \eta\right)$ is a section to $E$ (represented in the natural frame) we put

$$
\nabla \eta=d \eta-\eta \partial \log |\zeta|^{2}=\left(d \eta_{0}-\eta_{0} \partial \log |\zeta|, \ldots, d \eta_{n}-\eta_{n} \partial \log |\zeta|^{2}\right) .
$$

Then $\nabla$ is the canonical connection on $E$ endowed with the metric

$$
\|\eta\|^{2}=\sum\left|\eta_{j}\right|^{2} /|\zeta|^{2},
$$

and our curvature form is given by

$$
\Theta=\partial \bar{\partial} \log |s|^{2} .
$$

We now choose the sections $\eta, s, Q$ and the function $G(t)$ as follows

$$
\begin{array}{rlrl}
\eta_{j} & =\left(\zeta_{j}-z_{j}\right), & j=0, \ldots, n \\
s_{j} & =\frac{\partial \Phi}{\partial \zeta_{j}} /|\zeta|^{2}|z|^{2}, & \Phi=|\varsigma \wedge z|^{2} \quad \text { (sne sec. (1)) } \\
Q_{j} & =\frac{\partial \log |z|^{2}}{\partial z_{j}} & & \\
G(t) & =(1+t)^{N} & N \geq 0 .
\end{array}
$$

It is verified, as in section 1 , that $s$ and $\bar{\partial} Q$ take values in the subbundle $F$. Hence Proposition 14 applies.

We now claim that both kernels $K$ and $P$ are projective forms with respect to both variables $\zeta$ and $z$. First note that

$$
\left\langle s, \nabla^{\prime} \eta\right\rangle=\sum s_{j} d s_{j}-\sum s_{j} d z_{j}-\langle s, \zeta\rangle \partial \log |\zeta|^{2}
$$

since $\langle s, z\rangle=0$. Clearly this form is projective in $z$ and the connection is chosen so that it also becomes projective in $\zeta$. Hence the same holds for

$$
\bar{\partial}\left\langle s, \nabla^{\prime} \eta\right\rangle=\left\langle\bar{\partial} s, \nabla^{\prime} \eta\right\rangle+\langle s, \eta\rangle \Theta
$$


which implies $\left\langle\bar{\partial} s, \nabla^{\prime} \eta\right\rangle$ projective, since $\Theta$ is. In a similar way one shows that

$$
\left\langle\bar{\partial} Q, \nabla^{\prime} \eta\right\rangle
$$

is projective using the same argurment as in section 1 .

Now write

$$
K=\sum_{0}^{n} K_{p} \quad, \quad P=\sum_{0}^{n} P_{p}
$$

where $K_{p}$ and $P_{p}$ are the components of degree $p$ in $d z$. It is easily verified that $K_{p}$ and $P_{p}$ take values in $O(l)$ with

$$
l=p-N
$$

as forms in $z$ and in $O(-l)$ as forms in 5 . We can therefore consider $K_{p}$ and $P_{p}$ as kernels on $P^{n}$ acting on forms with values in $O(l)$. Let as before $K_{p, q}, P_{p, q}$ denote the components of bidegree $(p, q)$ in $z$.

Theorem 15. Let $f$ be a differential form on $\mathrm{P}^{n}$ of bidegree $(p, q)$ with values in $O(l), l \leq p$. Define $K$ and $P$ by (2) with $N=p-l$. Then

$$
f(z)=c_{n}\left\{\int_{p n} \bar{\partial} f \wedge K_{p, q}+(-1)^{p+q} \bar{\partial}_{z} \int_{p n} f \wedge K_{p, q}+(-1)^{p+q} \int_{p n} f \wedge P_{p, q}\right\} .
$$

This follows from Proposition 14 in the same way that Koppelmans formula is proved in $\mathrm{C}^{n}$. This is based on the formula

$$
\bar{\partial} K=c_{n}^{-1}[\Delta]+P
$$

(where $[\Delta]$ stands for the current "integration on the diagonal") and we already know that $\bar{\partial} K=P$ outside the diagonal $\Delta$. The fact that the singularity at the diagonal gives the same contribution as in the classical case follows since near the diagonal $K$ equals the classical Cauchy-Leray kernel modulo lower order terms.

Corollary.

$$
\begin{aligned}
& H^{p, q}\left(\mathrm{P}^{n}, 0\right)=0 . \text { for } p \neq q \\
& H^{p, p}\left(\mathrm{P}^{n}, 0\right) \approx c
\end{aligned}
$$

and $H^{p, p}$ is generated by $\left(\partial \bar{\partial} \log |\underline{z}|^{2}\right)^{p}$.

Proof: If $\bar{\partial} f=0$ the obstruction to solving

$$
\bar{\partial} g=f
$$


is given by the integral

$$
\int_{\mathbf{P} \frac{n}{s}} f(\zeta) \wedge P_{p, q}(\zeta, z)
$$

Since $l=0, N=p$. This implies that in the definition of $P$ the terms with $\beta_{1}>p$ vanish. Moreover the terms with $\beta_{1}<p$ cannot have degree $p$ in $d z$. Hence

$$
\begin{aligned}
P_{p} & =c\langle\bar{\partial} Q, d z\rangle^{p} \wedge \Theta^{n-p}= \\
& =c\left\langle\partial \bar{\partial} \log |z|^{2}\right)^{p} \wedge\left(\partial \bar{\partial} \log |\zeta|^{2}\right)^{n-p}
\end{aligned}
$$

which immediately gives the statement.

\section{References}

1. M. Andersson AND B. Berndtsson, Henkin-Ramirez formulas with weight factors, Ann Inst. Fourier 32 (1982).

2. S.G. GINDIKIN AND G.M. HENKIN, Integral geometry for $\bar{\partial}$-cohomo$\operatorname{logy}$ in $q$-linear concave domains in $\mathrm{P}^{n}$, Funcional Anal i Prilozen 12 (1978).

3. G.M.Henkin and P.L. Polyakov, Homotopy formulas for the $\bar{\partial}$ -operator on $\mathrm{p}^{\mathrm{n}}$ and the Radon-Penrose transform, Izv Akad Nauk SSSR 50 (1986).

4. J.P. Demailly and Ch. Laurent Thiebaur, Formules integrales pour les formes différentielles de type $(p, q)$ dans les varietés de Stein., Preprint (1986), Université de Paris VI.

Department of Mathematics

Chalmers University of Technology

and the University of Göteborg

S-412 96 Göteborg ${ }_{1}$ Sweden

Rebut el 14 de Gener de 1988 